\title{
LOEWY LENGTHS OF BLOCKS WITH ABELIAN DEFECT GROUPS
}

\author{
CHARLES W. EATON AND MICHAEL LIVESEY
}

(Communicated by Pham Huu Tiep)

\begin{abstract}
We consider $p$-blocks with abelian defect groups and in the first part prove a relationship between its Loewy length and that for blocks of normal subgroups of index $p$. Using this, we show that if $B$ is a 2-block of a finite group with abelian defect group $D \cong C_{2} a_{1} \times \cdots \times C_{2^{a_{r}}} \times\left(C_{2}\right)^{s}$, where $a_{i}>1$ for all $i$ and $r \geq 0$, then $d<\operatorname{LL}(B) \leq 2^{a_{1}}+\cdots+2^{a_{r}}+2 s-r+1$, where $|D|=2^{d}$. When $s=1$ the upper bound can be improved to $2^{a_{1}}+\cdots+$ $2^{a_{r}}+2-r$. Together these give sharp upper bounds for every isomorphism type of $D$. A consequence is that when $D$ is an abelian 2-group the Loewy length is bounded above by $|D|$ except when $D$ is a Klein-four group and $B$ is Morita equivalent to the principal block of $A_{5}$. We conjecture similar bounds for arbitrary primes and give evidence that it holds for principal 3-blocks.
\end{abstract}

\section{INTRODUCTION}

Let $G$ be a finite group and $k$ be an algebraically closed field of characteristic $p$. For a block $B$ of $k G$, write $\operatorname{LL}(B)$ for the Loewy length of $B$, that is, the smallest $n \in \mathbb{N}$ such that $\operatorname{Rad}(B)^{n}=0$ and write $e_{B}$ for the block idempotent corresponding to $B$. We are interested in upper and lower bounds for $\operatorname{LL}(B)$ in terms of the isomorphism type of the defect groups. For $p$-solvable groups it is proved in [10, p. 141] that the Loewy length is at most the order of a defect group and in 11] that it is strictly greater than $(p-1) d$, where $d$ is the defect of $B$. The upper bound does not hold when we remove the $p$-solvability hypothesis, as by [7, Theorem 2] the principal 2-block $B_{0}\left(k A_{5}\right)$ of $A_{5}$ (with Klein-four defect groups) has Loewy length 5 , although it is tempting to think that blocks Morita equivalent to $B_{0}\left(k A_{5}\right)$ are the only counterexamples, as we will see is indeed the case for $p=2$. As remarked in [14 the lower bound $(p-1) d$ also does not hold when we remove the $p$-solvability hypothesis, although $d$ could still be a lower bound. In this paper we restrict our attention to blocks with abelian defect groups, but investigate bounds on the Loewy length of a block for arbitrary finite groups.

Using the results of [6], which depends on the classification of finite simple groups, we prove our main result (see Theorem 4.1).

Theorem. Let $B$ be a 2-block with abelian defect group $D$. Suppose $D \cong C_{2^{a_{1}}} \times$ $\cdots \times C_{2^{a_{r}}} \times\left(C_{2}\right)^{s}$, where $a_{i}>1$ for all $i$ and $r \geq 0$. Write $|D|=2^{d}$.

Received by the editors July 29, 2016 and, in revised form, November 9, 2016 and November $24,2016$.

2010 Mathematics Subject Classification. Primary 20C20.

This research was supported by the EPSRC (grant no. EP/M015548/1). 
Then $d<\operatorname{LL}(B) \leq 2^{a_{1}}+\cdots+2^{a_{r}}+2 s-r+1$. If $s=1$, then $\operatorname{LL}(B) \leq$ $2^{a_{1}}+\cdots+2^{a_{r}}+2-r$.

The upper bounds in the above theorem are sharp for every isomorphism type of $D$ : take $B$ to be the principal block of $k\left(C_{2^{a_{1}}} \times \cdots \times C_{2^{a_{r}}} \times S L_{2}\left(2^{s}\right)\right)$ (see [1]). The lower bound was suggested in [14, where there is an excellent discussion of lower bounds on Loewy lengths. Note that for blocks of $p$-solvable groups with abelian defect groups, we have $\operatorname{LL}(B)=\operatorname{LL}(k D)$. We also note that in [19] it is proved that for principal 2-blocks with abelian defect groups, the Loewy length is bounded above by the maximum of $2 d+1$ and $|D|$.

A crucial element in establishing the above bounds is the consideration of the case where there is $N \triangleleft G$ such that $G=N D$. For $D$ elementary abelian we may apply the main result of 13 , which says that if $G$ is a split extension of $N$ (by a direct factor of $D$ ), then $B$ behaves as if $N$ were a direct factor of $G$. Theorem 2.1 generalizes this result and we use it to show how for arbitrary abelian $D$ we may compare the Loewy lengths of $B$ and the block of $N$ covered by $B$. This works for all primes, and we hope Theorem 2.1 is of wider interest.

The paper is structured as follows. In Section 2 we give the generalization of the theorem of [13] and the theorem concerning Loewy length of blocks when there is a normal subgroup of index $p$. In Section 3 we give some preliminary results needed for the proof of the bounds, which we give in Section 4. In Section 5 we consider similar bounds for odd primes and make a conjecture.

\section{Normal SUbGroups OF INDEX $p$}

We first prove a generalization of a theorem of Koshitani and Külshammer [13].

Theorem 2.1. Let $G$ be a finite group and $B$ a block of $k G$ with abelian defect group $D$ with primary decomposition $D=D_{1} \times \cdots \times D_{t}$. Let $N \triangleleft G$ such that $G=N D_{r}$ for some $r \leq t, D_{j} \leq N$ for all $j \neq r$ and $[G: N]=p$. Let $b$ be $a$ block of $k N$ covered by $B$. Then $e_{B}=e_{b}$ and there exists an element $a \in Z(B)$ of multiplicative order dividing $\left|D_{r}\right|$ such that $B=\bigoplus_{j=0}^{p-1} a^{j} k N e_{b}$.

Proof. We follow the proof of [13], where it is assumed that $D_{r} \cap N=1$. Let $\mathcal{A}$ be a root of $B$ in $C_{G}(D)$ and $N_{G}(D)_{\mathcal{A}}$ the stabilizer of $e_{\mathcal{A}}$ in $N_{G}(D)$. As in [13, $e_{B}=e_{b}, Z(B)$ is a crossed product of $Z(b)$ over $G / N$ and $N_{G}(D)_{\mathcal{A}} / C_{G}(D)$ is a $p^{\prime}$-group.

Now $N_{G}(D)_{\mathcal{A}} / C_{G}(D)$ acts on both $D$ and $D \cap N$ by conjugation. Consider the subgroup $Q \leq D$ generated by $D_{r}$ and all its $N_{G}(D)_{\mathcal{A}}$-conjugates. This group has exponent $\left|D_{r}\right|$ and so by [9, $\S 5$, Theorem 2.2] $Q=Q_{1} \times Q_{2}$, where $Q_{1}$ and $Q_{2}$ are $N_{G}(D)_{\mathcal{A}}$-invariant, $Q_{1}$ is homocyclic of exponent $\left|D_{r}\right|$ and $Q_{2}$ has exponent strictly less than $\left|D_{r}\right|$. As $\left[Q_{1}: Q_{1} \cap N\right]=p$ we have $Q_{1} \cap N \cong R_{1} \times R_{2}$, where $R_{1}$ is cyclic of order $\left|D_{r}\right| / p$ and $R_{2}$ is homocyclic of exponent $\left|D_{r}\right|$. Again by [9, $\S 5$, Theorem 2.2] we can assume $R_{1}$ and $R_{2}$ are both $N_{G}(D)_{\mathcal{A}}$-invariant and therefore by [9, $\S 3$, Theorem 3.2] $R_{2}$ must have an $N_{G}(D)_{\mathcal{A}}$-invariant complement $R$ in $Q_{1}$. So $R$ is cyclic, $N_{G}(D)_{\mathcal{A}}$-invariant, $|R|=\left|D_{r}\right|$ and $[R: R \cap N]=p$.

Now $|\operatorname{Aut}(R)|_{p^{\prime}}=p-1$ and the natural homomorphism $\operatorname{Aut}(R) \rightarrow \operatorname{Aut}(R /(R \cap$ $N)$ ) is surjective and so any non-trivial $p^{\prime}$-element of $\operatorname{Aut}(R)$ acts non-trivially on $R /(R \cap N)$. However, $\left[R, N_{G}(D)_{\mathcal{A}}\right] \leq R \cap N$ and so every element of $N_{G}(D)_{\mathcal{A}}$ acts trivially on $R /(R \cap N)$. Therefore, $N_{G}(D)_{\mathcal{A}}$ commutes with $R$. 
Let $\alpha=\mathcal{A}^{C_{G}\left(C_{D}\left(N_{G}(D)_{\mathcal{A}}\right)\right)}$. By Watanabe's result [22, Theorem 2(ii)], the map

$$
\begin{aligned}
f: Z(B) & \rightarrow Z(\alpha), \\
z & \mapsto \operatorname{Br}_{C_{D}\left(N_{G}(D)_{\mathcal{A}}\right)}(z) e_{\alpha}
\end{aligned}
$$

is an isomorphism of $k$-algebras. Note that since $R$ commutes with $N_{G}(D)_{\mathcal{A}}$ we have that $R \leq Z\left(C_{G}\left(C_{D}\left(N_{G}(D)_{\mathcal{A}}\right)\right)\right)$ and also $C_{G}\left(C_{D}\left(N_{G}(D)_{\mathcal{A}}\right)\right)=R C_{N}\left(C_{D}\left(N_{G}(D)_{\mathcal{A}}\right)\right)$. Therefore $\alpha$ and hence $Z(\alpha)$ are $G / N$-graded. Recall that $Z(B)$ is also $G / N$-graded and since $e_{\alpha} \in k N, f$ respects these gradings. Setting $a:=f^{-1}\left(e_{\alpha} g\right)$, where $R=\langle g\rangle$, proves the theorem.

We now use Theorem 2.1 to prove upper and lower bounds on the Loewy length of $B$ in terms of the Loewy length of $b$. Note that using [5, Theorem 3.4(I)] one can derive an upper bound of $p \mathrm{LL}(b)$. However, we require a much more precise bound.

Corollary 2.2. Assume we are in the setting of Theorem 2.1. Then

(i) $\operatorname{Rad}(B)=\operatorname{Rad}(b) Z(B)+(1-a) B$;

(ii) $\operatorname{LL}(b)+(p-1) \leq \mathrm{LL}(B) \leq \mathrm{LL}(b)+\left|D_{r}\right|-\left|D_{r}\right| / p$.

Proof. (i) Certainly $\operatorname{Rad}(b) Z(B)$ and $(1-a) B$ are nilpotent ideals of $B$ and so

$$
\operatorname{Rad}(B) \supseteq \operatorname{Rad}(b) Z(B)+(1-a) B .
$$

Then

$$
B /(\operatorname{Rad}(b) Z(B))=B /(\operatorname{Rad}(b) B) \cong(b / \operatorname{Rad}(b)) \otimes_{b} B \cong(b / \operatorname{Rad}(b)) \otimes_{k} k C_{p},
$$

where the last isomorphism is given by $(x \otimes a) \mapsto(x \otimes g)$, where $C_{p}=\langle g\rangle$. Therefore

$$
\operatorname{Rad}(B) /(\operatorname{Rad}(b) Z(B)+(1-a) B)
$$

is semisimple and so $\operatorname{Rad}(B)=\operatorname{Rad}(b) Z(B)+(1-a) B$.

(ii) If $\gamma_{1}, \ldots, \gamma_{s} \in \operatorname{Rad}(b)$ with $\gamma_{1} \ldots \gamma_{s} \neq 0$, then $\gamma_{1} \ldots \gamma_{s}(1-a)^{(p-1)}$ is a nonzero product of elements of $\operatorname{Rad}(B)$, and so $\operatorname{LL}(B) \geq \operatorname{LL}(b)+(p-1)$.

Next suppose $\beta_{1}, \ldots, \beta_{s} \in \operatorname{Rad}(B)$ with $\beta_{1} \ldots \beta_{s} \neq 0$ with $\beta_{i}=\operatorname{Rad}(B)$. Then by (i) there exist $\gamma_{1}, \ldots, \gamma_{s}$ such that $\gamma_{1} \ldots \gamma_{s} \neq 0$ and each $\gamma_{i}$ is either $(1-a)$ or is an element of $\operatorname{Rad}(b)$. Say $t$ of the $\gamma_{i}$ 's are $(1-a)$ 's. Then clearly $t<$ $\left|D_{r}\right|$ and since $(1-a)^{p} \in \operatorname{Rad}(b)$ we also have that $\lfloor t / p\rfloor+s-t<\operatorname{LL}(b)$. As increasing $t$ can only decrease the left hand side of this inequality, it is also true that $\left\lfloor\left(\left|D_{r}\right|-1\right) / p\right\rfloor+s-\left(\left|D_{r}\right|-1\right)<\operatorname{LL}(b)$ and so $\left|D_{r}\right| / p-1+s-\left(\left|D_{r}\right|-1\right)<\operatorname{LL}(b)$ giving that $s<\mathrm{LL}(b)+\left|D_{r}\right|-\left|D_{r}\right| / p$ and the theorem is proved.

\section{FURThER PRELIMINARY RESUlts}

The following result is well known, but we are not aware of an explicit reference.

Lemma 3.1. Let $G$ be a finite group and $B$ be a p-block of $G$ with defect group $D$. Let $N \triangleleft G$ and let $b$ be a block of $N$ covered by $B$. If $D \leq N$, then $\operatorname{LL}(B)=\operatorname{LL}(b)$.

Proof. The proof given in [15, Lemma 4.1] for the case $G / N$ is a $p^{\prime}$-group carries through in the general situation, but we include a simple argument suggested by Markus Linckelmann.

Let $I_{G}(b)$ be the stabilizer of $b$ in $G$. Since there is a block of $I_{G}(b)$ covering $b$ and Morita equivalent to $B$, we may assume that $G=I_{G}(b)$. Then $\operatorname{Rad}(b) B \subseteq \operatorname{Rad}(B)$. Since $D \leq N$, every $B$-module is relatively $N$-projective, and so in particular 
$B / \operatorname{Rad}(b) B$ and every quotient module is. Now the restriction of $B / \operatorname{Rad}(b) B$ to $k N$ is a semisimple $b$-module, so it follows that $B / \operatorname{Rad}(b) B$ is semisimple as a $B$-module. Hence $\operatorname{Rad}(B) \subseteq \operatorname{Rad}(b) B$, and so $\operatorname{Rad}(B)=\operatorname{Rad}(b) B$. Again since $b$ is $G$-stable, we have $\operatorname{LL}(B)=\operatorname{LL}(b)$.

Lemma 3.2. Let $G_{1}, \ldots, G_{t}$ be finite groups and $G=G_{1} \times \cdots \times G_{t}$. For each $i$ let $B_{i}$ be a block of $G_{i}$ and let $B=B_{1} \otimes \cdots \otimes B_{s}$ be the block of $G$ covering each $B_{i}$. Let $D_{i}$ be a defect group of $B_{i}$, so $D=D_{1} \times \cdots \times D_{t}$ is a defect group of $B$. Then $\operatorname{LL}(B)=\left(\sum_{i=1}^{t} \operatorname{LL}\left(B_{i}\right)\right)-t+1$.

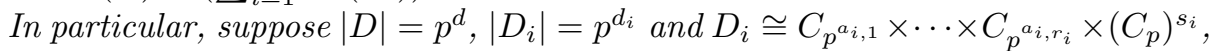
where each $a_{i, j}>1$. If $d_{i}<\operatorname{LL}\left(B_{i}\right) \leq p^{a_{i, 1}}+\cdots+p^{a_{i, r_{i}}}+p s_{i}-r_{i}+1$ for each $i$, then $d<\operatorname{LL}(B) \leq \sum_{i=1}^{t} \sum_{j=1}^{r_{i}} p^{a_{i, j}}+p \sum_{i=1}^{t} s_{i}-\left(\sum_{i=1}^{t} r_{i}\right)+1$. If, in addition, $\operatorname{LL}\left(B_{i}\right) \leq p^{a_{i, 1}}+\cdots+p^{a_{i, r_{i}}}+p s_{i}-r_{i}$ for at least one $i$, then $\operatorname{LL}(B) \leq$ $\sum_{i=1}^{t} \sum_{j=1}^{r_{i}} p^{a_{i, j}}+p \sum_{i=1}^{t} s_{i}-\left(\sum_{i=1}^{t} r_{i}\right)$.

Proof. The first part is [3, 1.1] and the second part is just a direct application of the first.

Before proceeding we recall the definition and some properties of the generalized Fitting subgroup $F^{*}(G)$ of a finite group $G$. Details may be found in [4].

A component of $G$ is a subnormal quasisimple subgroup of $G$. The components of $G$ commute, and we define the layer $E(G)$ of $G$ to be the normal subgroup of $G$ generated by the components. It is a central product of the components. The Fitting subgroup $F(G)$ is the largest nilpotent normal subgroup of $G$, and this is the direct product of $O_{r}(G)$ for all primes $r$ dividing $|G|$. The generalized Fitting subgroup $F^{*}(G)$ is $E(G) F(G)$. A crucial property of $F^{*}(G)$ is that $C_{G}\left(F^{*}(G)\right) \leq$ $F^{*}(G)$, so in particular $G / F^{*}(G)$ may be viewed as a subgroup of $\operatorname{Aut}\left(F^{*}(G)\right)$.

Lemma 3.3. Let $B$ be a block of a finite group $G$ with abelian defect group $D$. Then there is a group $H$ and a block $C$ of $H$ with defect group $D_{C} \cong D$ such that $\mathrm{LL}(C)=\mathrm{LL}(B)$ and the following are satisfied:

(i) $C$ is quasiprimitive, that is, for every normal subgroup $N$ of $H$, every block of $N$ covered by $C$ is $H$-stable.

(ii) $H$ is generated by the defect groups of $C$.

(iii) If $N \triangleleft H$ and $C$ covers a nilpotent block of $N$, then $N \leq Z(H) O_{p}(H)$. In particular, $O_{p^{\prime}}(H) \leq Z(H)$.

(iv) $O_{p}(H) \leq Z(H)$.

(v) Every component of $H$ is normal in $H$.

(vi) If $B$ is the principal block of $G$, then $C$ is the principal block of $H$.

Proof. Consider pairs $\left(\left[G: O_{p^{\prime}}(Z(G))\right],|G|\right)$ with the lexigraphic ordering. There are three processes, labelled (a), (b), (c), which will be applied repeatedly and in various combinations. We describe these processes and show that they strictly reduce $\left(\left[G: O_{p^{\prime}}(Z(G))\right],|G|\right)$ when applied non-trivially, so that repeated application of (a), (b) and (c) terminates.

(a) Let $N \triangleleft G$ and let $b$ be a block of $N$ covered by $B$. Write $I=I_{G}(b)$ for the stabilizer of $b$ in $G$, and $B_{I}$ for the Fong-Reynolds correspondent. Now $B_{I}$ is Morita equivalent to $B$ and they have isomorphic defect groups. Clearly $O_{p^{\prime}}(Z(G)) \leq O_{p^{\prime}}(Z(I))$, and if $I \neq G$, then $\left[I: O_{p^{\prime}}(Z(I))\right]<\left[G: O_{p^{\prime}}(Z(G))\right]$. Process (a) involves replacing $B$ by $B_{I}$. 
(b) is the replacement of $G$ by $N=\left\langle D^{g}: g \in G\right\rangle$ and $B$ by any block $b$ of $N$ covered by $B$, as in Lemma 3.1. In this case $\left[N: O_{p^{\prime}}(Z(N))\right] \leq\left[G: O_{p^{\prime}}(Z(G))\right]$ and $|N|<|G|$ if $N \neq G$. Since defect groups of $b$ are intersections of defect groups of $B$ with $N$ (see [2, 15.1]), it follows that $B$ and $b$ share a defect group.

(c) Let $N \triangleleft G$ and suppose that $B$ covers a nilpotent block $N$ of $G$ such that $N \not \leq Z(G) O_{p}(G)$. Let $b^{\prime}$ be a block of $Z(G) N$ covered by $B$ and covering $b$. By performing (a) first we may assume that $b^{\prime}$ is $G$-stable. Further $b^{\prime}$ must also be nilpotent. Using the results of [17, as outlined in [6, Proposition 2.2], $B$ is Morita equivalent to a block $\tilde{B}$ of a central extension $\tilde{L}$ of a finite group $L$ by a $p^{\prime}$-group such that there is an $M \triangleleft L$ with $M \cong D \cap(Z(G) N), G / Z(G) N \cong L / M$, and $\tilde{B}$ has defect group isomorphic to $D$. Note that $\left[\tilde{L}: O_{p^{\prime}}(Z(\tilde{L}))\right] \leq|L|=[G$ : $Z(G) N]|D \cap(Z(G) N)|<\left[G: O_{p^{\prime}}(Z(G))\right]$ and that $M \leq O_{p}(\tilde{L}) Z(\tilde{L})$. Process $(\mathrm{c})$ consists of replacing $G$ by $\tilde{L}$ and $B$ by $\tilde{B}$.

Repeated application of (a), (b) and (c) must eventually terminate, in which case we are left with $H$ and a block $C$ of $H$ with defect group $D_{C} \cong D$ satisfying conditions (i)-(iii).

To see that $H$ and $C$ satisfy (iv), note that $D \leq C_{G}\left(O_{p}(G)\right) \triangleleft G$, so (iv) is a consequence of (ii).

(v) Write $L_{1}, \ldots, L_{m}$ for the components of $G$. We may assume that $B$ is quasiprimitive, $G$ is generated by the defect groups of $B$ and that $B$ does not cover any nilpotent block with non-central defect groups. As above the generalized Fitting subgroup $F^{*}(G)=E(G) F(G)$, where $E(G)=L_{1} \cdots L_{m} \triangleleft G$. Since by (iv) we may assume $O_{p}(G) \leq Z(G)$ and by (iii) we may assume $O_{r}(G) \leq Z(G)$ for primes $r \neq p$, we assume that $F^{*}(G)=E(G) Z(G)$. By a similar argument we also have that $Z\left(F^{*}(G)\right)=Z(G)$. Let $\varphi: G \rightarrow S_{m}$ be the homomorphism given by the permutation action on the components. Now $D \cap F^{*}(G)$ is a defect group for a (non-nilpotent) block of $F^{*}(G)$ covered by $B$. Hence $\left(D \cap F^{*}(G)\right) / O_{p}(Z(G))$ is a defect group for a block of $F^{*}(G) / O_{p}(Z(G))$. Therefore $\left(D \cap F^{*}(G)\right) / O_{p}(Z(G))$ is a radical $p$-subgroup of $F^{*}(G) / O_{p}(Z(G)$ ) (recall that a $p$-subgroup $Q$ of a finite group of a finite group $H$ is radical if $Q=O_{p}\left(N_{G}(Q)\right)$ and that defect groups are radical p-subgroups) and so $\left(D \cap F^{*}(G)\right) Z(G) / Z(G)$ is a radical $p$-subgroup of $F^{*}(G) / Z(G) \cong\left(L_{1} Z(G) / Z(G)\right) \times \cdots \times\left(L_{m} Z(G) / Z(G)\right)$. Note that $\left(D \cap F^{*}(G)\right) Z(G) / Z(G)$ is not necessarily a defect group, hence our move to the weaker condition of being a radical $p$-subgroup. By [20, Lemma 2.2] we have therefore $\left(D \cap F^{*}(G)\right) Z(G) / Z(G)=D_{1} \times \cdots \times D_{m}$, where $D_{i}=$ $\left(D \cap F^{*}(G)\right) Z(G) / Z(G) \cap\left(L_{i} Z(G) / Z(G)\right)$. Since $B$ is quasiprimitive it follows that each $D_{i}$ is non-trivial (otherwise $B$ covers a nilpotent block of a non-central normal subgroup, namely the subgroup generated by $Z(G)$ and the orbit of $L_{i}$ ). Hence since $D$ is abelian we have $D \leq \operatorname{ker}(\varphi)$, and so $(\mathrm{v})$ follows since $G$ is generated by the conjugates of $D$.

To prove (vi), it suffices to show that the processes (a), (b) and (c) respect principal blocks. If $N \triangleleft G$ and $B$ is principal, then $B$ covers the principal block of $N$. Also, the Fong-Reynolds correspondence takes principal blocks to principal blocks. It follows that if $B$ is the principal block of $G$, then the block constructed in (a) and (b) may be taken to be the principal block. In (c), if $B$ is the principal block and $B$ covers a nilpotent block $b$ of $N \triangleleft G$, then $b$ is the principal block of $N$ and by a theorem of Frobenius (see [9, 7.4.5]) we have that $N$ has a normal $p$-complement. We may then apply the reduction to $O_{p^{\prime}}(N) \triangleleft G$. But $B$ covers the 
principal block of $O_{p^{\prime}}(N)$, i.e., $O_{p^{\prime}}(N)$ lies in the kernel of $B$, and we may replace $B$ by the principal block of $G / O_{p^{\prime}}(N)$.

Proposition 3.4 ([6, Theorem 6.1]). Let $k$ be an algebraically closed field of characteristic $2, G$ a quasisimple group and $B$ be a block of $k G$ with abelian defect group $P$, then one (or more) of the following holds:

(i) $G / Z(G)$ is one of $A_{1}\left(2^{a}\right),{ }^{2} G_{2}(q)$ (where $a>1$ and $q \geq 27$ is a power of 3 with odd exponent), or $J_{1}, B$ is the principal block and $P$ is elementary abelian.

(ii) $G$ is $\mathrm{Co}_{3}, B$ is a non-principal block, $P \cong C_{2} \times C_{2} \times C_{2}$.

(iii) There exists a finite group $\tilde{G}$ such that $G \unlhd \tilde{G}, Z(G) \leq Z(\tilde{G})$ and such that $B$ is covered by a nilpotent block of $\tilde{G}$.

(iv) $B$ is Morita equivalent to a block $C$ of $k L$ where $L=L_{0} \times L_{1}$ is a subgroup of $G$ such that the following holds: The defect groups of $C$ are isomorphic to $P, L_{0}$ is abelian and the block of $k L_{1}$ covered by $C$ has Klein-four defect groups.

\section{Proof of the MAIN RESUlT}

Theorem 4.1. Let $B$ be a 2-block with abelian defect group $D$. Suppose $D \cong$ $C_{2^{a_{1}}} \times \cdots \times C_{2^{a_{r}}} \times\left(C_{2}\right)^{s}$, where $a_{i}>1$ for all $i$ and $r \geq 0$. Write $|D|=2^{d}$.

Then $d<\operatorname{LL}(B) \leq 2^{a_{1}}+\cdots+2^{a_{r}}+2 s-r+1$. If $s=1$, then $\operatorname{LL}(B) \leq$ $2^{a_{1}}+\cdots+2^{a_{r}}+2-r$.

Proof. We may assume that $B$ satisfies conditions (i) to (v) of Lemma 3.3 .

Next suppose that $N$ is a normal subgroup of $G$ of index 2 and $b$ is a block of $N$ covered by $B$. Since $B$ is quasiprimitive $G$ stabilizes $b$, so by $[2, \S 15$, Theorem 1 (4)] there exists a block $B^{*}$ of $G$ covering $b$ with defect group $D^{*}$ such that $D^{*} N=G$. But since $G / N$ is a 2-group $B$ is the unique block of $G$ covering $b$, so $B^{*}=B$. We can now apply Corollary 2.2(ii), so that it suffices to prove the Theorem for $b$. By repeated application of this, and possibly further application of Lemma 3.3, we may assume that $G=O^{2}(G)$.

By the arguments in Lemma 3.3(v) and the fact that generalized Fitting subgroups are self-centralizing we may assume that

$$
S_{1} \times \cdots \times S_{m} \leq G / Z(G) \leq \operatorname{Aut}\left(S_{1}\right) \times \cdots \operatorname{Aut}\left(S_{m}\right) \leq \operatorname{Aut}\left(S_{1} \times \cdots \times S_{m}\right)
$$

for normal non-abelian simple groups $S_{1}, \ldots, S_{m}$. Continuing with the notation from Lemma 3.3. (v) we have $L_{i} Z(G) / Z(G) \cong S_{i}$ and $L_{i} \triangleleft G$ for each $i$. By the Schreier conjecture $G / E(G)$ is solvable, and hence, as we are assuming both $G=$ $O^{2}(G)$ and $G=O^{2^{\prime}}(G)$, we may assume that $G=E(G)$.

The remainder of the proof proceeds almost as in that of [6, Theorem 8.3]. There it is proved that we may assume (by replacing $B$ by a Morita equivalent block of another finite group if necessary) that $G=E / Z$, where $E=U \times V \times W$ with $Z \leq O_{2}(Z(E)) \cap U, B$ is a product of blocks $B_{U / Z}, B_{V}$ and $B_{W}$ of $U / Z, V$ and $W$ respectively and $D=D_{U / Z} \times D_{V} \times D_{W}$ with the following properties: $B_{U / Z}$ is a block of $U / Z$ with defect group $D_{U / Z}$ and is Morita equivalent to its Brauer correspondent in $N_{U / Z}\left(D_{U / Z}\right) ; V=V_{1} \times \cdots \times V_{t}$ and $B_{V}$ is a block of $V$ with defect group $D_{V}$ and which is a product of blocks $B_{V_{i}}$ of $V_{i}$ with Klein-four defect groups; $W=W_{1} \times \cdots \times W_{u}$ and $B_{W}$ is a block of $W$ with defect group $D_{W}$ and which is a product of blocks $B_{W_{i}}$ of simple groups $W_{i}$ satisfying condition (i) or (ii) of Proposition 3.4 
By Lemma $3.1 \mathrm{LL}\left(B_{U / Z}\right)=\mathrm{LL}\left(k D_{U / Z}\right)$, and so $B_{U / Z}$ satisfies the required inequality. Since a block with Klein-four defect groups has Loewy length 3 or 5 , by Lemma $3.2 B_{V}$ also satisfies the inequality. It remains to consider the blocks satisfying (i) or (ii) of Proposition [3.4. By [1] the Loewy length of the principal block of $S L_{2}\left(2^{a}\right)$ is $2 a+1$. By [19, Theorem 4.1], for all $m$ the principal 2-block of ${ }^{2} G_{2}\left(3^{2 m+1}\right)$, with elementary abelian defect group of order 8 , has Loewy length 7. Now consider the principal block of the smallest Janko group $J_{1}$, which also has elementary abelian defect group of order 8 . The structure of the principal indecomposable modules has been determined in this case in [19], although we note that there is a mistake there in the structure of $P_{76}$ which does not alter the Loewy length, which is 7. By [16, 1.5] the principal block of $\mathrm{Co}_{3}$ is Morita equivalent to that of $\operatorname{Aut}\left(S L_{2}(8)\right)$. Since $\left[\operatorname{Aut}\left(S L_{2}(8)\right): S L_{2}(8)\right]=3$, Lemma 3.1 tells us the Loewy length is that of the principal block of $S L_{2}(8)$, i.e., 7 , where the defect group is elementary abelian of order 8 .

Next we note that if $s=1$, then $V=\{1\}$ and the relevant block $\left(B_{U / Z}\right.$ or $B_{W}$, depending on whether $D_{U / Z}$ or $D_{W}$ has a factor isomorphic to $C_{2}$ ) satisfies the stronger upper bound.

The result then follows by Lemma 3.2 .

Corollary 4.2. Let $B$ be a 2-block with abelian defect group $D$. Then $\operatorname{LL}(B) \leq|D|$ unless $D$ is a Klein-four group.

\section{OTHER PRIMES}

There is relatively little evidence for similar bounds for odd primes, but it is tempting to conjecture the following:

Conjecture 5.1. Let $B$ be a block of a finite group $G$ with abelian defect group $D \cong C_{p^{a_{1}}} \times \cdots \times C_{p^{a_{r}}} \times\left(C_{p}\right)^{s}$, where $a_{i}>1$ for all $i$ and $r \geq 0$. Write $|D|=p^{d}$. Then

$$
d<\mathrm{LL}(B) \leq p^{a_{1}}+\cdots+p^{a_{r}}+p s-r+\delta,
$$

where $\delta=1$ if $s$ is even and $\delta=0$ if $s$ is odd.

Note that when $D$ is cyclic $\operatorname{LL}(B)$ may be computed from the Brauer tree, and is bounded above by $\left(\frac{|D|-1}{e(B)}\right) e(B)+1=|D|$, where $e(B)$ is the number of simple $B$-modules (here $\frac{|D|-1}{e(B)}$ is the multiplicity of an exceptional vertex and $e(B)$ is an upper bound for the number of edges emanating from a vertex). By [14, 2.8] $\mathrm{LL}(B) \geq \frac{|D|-1}{e(B)}+1>d$ since $e(B) \leq p-1$.

We discuss the case $p=3$ below, but mention now that the conjectured bound is achieved in this case by taking the principal block of $C_{3^{a_{1}}} \times \cdots \times C_{3^{a_{r}}} \times\left(M_{23}\right)^{s / 2}$ if $s$ is even or $C_{3^{a_{1}}} \times \cdots \times C_{3^{a_{r}}} \times\left(M_{23}\right)^{(s-1) / 2} \times C_{3}$ if $s$ is odd, where $M_{23}$ is the sporadic simple group of that name.

A key special case of Conjecture 5.1 is where $D \cong C_{p} \times C_{p}$. There is a relative scarcity of computed examples when $p>3$, and we do not know of any examples in this case which make the upper bound sharp for $s>1$. Hence we ask the following:

Question 5.2. Is there always a block $B$ of some finite group $G$ with defect group $C_{p} \times C_{p}$ and $\mathrm{LL}(B)=2 p+1$ ?

In 12 Koshitani describes the finite groups whose Sylow 3-subgroups are abelian, and in [15] it is shown that any principal 3-block with abelian defect groups of order 
$3^{2}$ has Loewy length 5 or 7 . Based on this we show the following. Note that at present it is not realistic to calculate the Loewy length for the principal block of the sporadic simple group $O^{\prime} N$ (with elementary abelian Sylow 3-subgroups of order $3^{4}$ ) by computer or otherwise. Conjecture 5.1 predicts that the Loewy length lies between 5 and 13 .

Proposition 5.3. Suppose that the Loewy length of the principal 3-block of $O^{\prime} N$ is at least 5 and at most 13 . Let $G$ be a finite group with Sylow 3 -subgroup $D \cong$ $C_{3^{a}} \times\left(C_{3}\right)^{s}$, where $a>1$, and let $B$ be the principal 3-block of $G$. Write $d$ for the defect of $B$. Then

$$
d<\operatorname{LL}(B) \leq 3^{a}+3 s-1+\delta,
$$

where $\delta=1$ if $s$ is even and $\delta=0$ if $s$ is odd.

Proof. We may assume that $B$ satisfies conditions (i) to (vi) of Lemma 3.3, Since $O_{3^{\prime}}(G)$ is in the kernel of the principal block, we may further assume that $O_{3^{\prime}}(G)=$ 1. By [12, which is based on [8],

$$
G=O_{3}(G) \times G_{1} \times \cdots \times G_{n}
$$

where $n \geq 0$ and each $G_{i}$ is either a non-abelian simple group with cyclic Sylow 3 -subgroups or is one of the following (taking the notational convention that the unitary group $G U_{n}(q)$ is defined as a subgroup of $\left.G L_{n}\left(q^{2}\right)\right)$ :

(i) $A_{6}, A_{7}, A_{8}, M_{22}, M_{23}, H S, O^{\prime} N$;

(ii) $P S L_{3}(q)$ where $3 \mid q-1$ but $3^{2} \not q-1$;

(iii) $P S U_{3}(q)$ where $3 \mid q+1$ but $3^{2} \not q+1$;

(iv) $\operatorname{PSp}_{4}(q)$ where $3 \mid q-1$;

(v) $P S p_{4}(q)$ where $q>2$ and $3 \mid q+1$;

(vi) $P S L_{4}(q)$ where $q>2$ and $3 \mid q+1$;

(vii) $P S U_{4}(q)$ where $3 \mid q-1$;

(viii) $P S L_{5}(q)$ where $3 \mid q+1$;

(ix) $P S U_{5}(q)$ where $3 \mid q-1$;

(x) $P S L_{2}\left(3^{m}\right)$ where $m \geq 2$.

We first show that $d<\operatorname{LL}(B) \leq 3^{a}+3 s$, and then consider what happens when we further assume that $s$ is odd later. By Lemma 3.2, in order to show that $d<\operatorname{LL}(B) \leq 3^{a}+3 s$ it suffices to check that this inequality holds for each factor group in turn. We have treated the case that $D$ is cyclic above, so it suffices to consider the cases (i)-(x). 7 .

(i)-(iii) In all cases except $O^{\prime} N$ we have $D \cong C_{3} \times C_{3}$ and by 15 . $\mathrm{LL}(B)=5$ or

(iv)-(ix) In these cases we claim the Sylow 3-subgroups are isomorphic to $C_{3^{r}} \times$ $C_{3^{r}}$ for some $r \geq 1$. First note that by calculating orders we have that the Sylow 3 -subgroups all have order $3^{2 r}$, where $3^{r}$ is the maximum power of 3 dividing $q-1$ in the cases (iv), (vii) and (ix) or the maximum power of 3 dividing $q+1$ in the cases (v), (vi) and (viii). We next construct the 3 -subgroups

(iv) $C_{3^{r}} \times C_{3^{r}} \leq \mathbb{F}_{q}^{\times} \times \mathbb{F}_{q}^{\times} \leq S p_{2}(q) \times S p_{2}(q) \leq S p_{4}(q)$;

(v) $C_{3^{r}} \times C_{3^{r}} \leq\left(\mathbb{F}_{q^{2}}^{\times}\right)^{q-1} \times\left(\mathbb{F}_{q^{2}}^{\times}\right)^{q-1} \leq S p_{2}(q) \times S p_{2}(q) \leq S p_{4}(q)$;

(vi) $C_{3^{r}} \times C_{3^{r}} \leq \mathbb{F}_{q^{2}}^{\times} \times \mathbb{F}_{q^{2}}^{\times} \leq G L_{2}(q) \times G L_{2}(q) \leq G L_{4}(q)$;

(vii) $C_{3^{r}} \times C_{3^{r}} \leq \mathbb{F}_{q}^{\times} \times \mathbb{F}_{q}^{\times} \leq G L_{1}\left(q^{2}\right) \times G L_{1}\left(q^{2}\right) \leq G U_{4}(q)$;

(viii) $C_{3^{r}} \times C_{3^{r}} \leq \mathbb{F}_{q^{2}}^{\times} \times \mathbb{F}_{q^{2}}^{\times} \leq G L_{2}(q) \times G L_{2}(q) \times G L_{1}(q) \leq G L_{5}(q)$;

(ix) $C_{3^{r}} \times C_{3^{r}} \leq \mathbb{F}_{q}^{\times} \times \mathbb{F}_{q}^{\times} \leq G L_{1}\left(q^{2}\right) \times G L_{1}\left(q^{2}\right) \times G U_{1}(q) \leq G U_{5}(q)$. 
Note that all these subgroups survive in the simple subquotients and so $D \cong$ $C_{3^{r}} \times C_{3^{r}}$ and therefore we in fact have $D \cong C_{3} \times C_{3}$ and as in cases (i)-(iii) $\mathrm{LL}(B)=5$ or 7 .

(x) By [3] $\operatorname{LL}\left(B_{0}\left(P S L_{2}\left(3^{m}\right)\right)\right)=2 m+1$ and we are done.

Now suppose further that $s$ is odd. Then some direct factor of $G$ has Sylow 3-subgroups $\left(C_{3}\right)^{r}$ for odd $r$, in which case that factor must either be $C_{3}$ itself or $P S L_{2}\left(3^{r}\right)$. In either case by Lemma 3.2 we have $\operatorname{LL}(B) \leq 3^{a}+3 s-1$.

Finally we prove the result concerning blocks of $p$-solvable groups with abelian defect groups mentioned in the introduction, which in particular implies that such blocks satisfy sharper bounds than in general. Note that this does not use the classification of finite simple groups.

Proposition 5.4. Let $B$ be a block of a p-solvable group $G$ with abelian defect group $D$. Then $\operatorname{LL}(B)=\operatorname{LL}(k D)$.

Proof. By Lemma 3.3 we may assume that $O_{p^{\prime}}(G) \leq Z(G)$ and $C_{G}\left(O_{p}(G)\right)=G$, so by [9, 6.3.2] $G=C_{G}\left(O_{p}(G)\right) \leq O_{p}(G) Z(G)$ and the result follows.

\section{ACKNOWLEDGMENTS}

We thank Burkhard Külshammer for suggesting the application of [6] to the determination of Loewy lengths. We also thank the referee for helpful comments. We thank Naoko Kunigi for spotting an error in an earlier version of the paper.

\section{REFERENCES}

[1] J. L. Alperin, Projective modules for SL(2, $\left.2^{n}\right)$, J. Pure Appl. Algebra 15 (1979), no. 3, 219-234, DOI 10.1016/0022-4049(79)90017-3. MR.537496

[2] J. L. Alperin, Local representation theory, Cambridge Studies in Advanced Mathematics, vol. 11, Cambridge University Press, Cambridge, 1986. Modular representations as an introduction to the local representation theory of finite groups. MR860771

[3] Henning Haahr Andersen, Jens Jørgensen, and Peter Landrock, The projective indecomposable modules of SL $\left(2, p^{n}\right)$, Proc. London Math. Soc. (3) 46 (1983), no. 1, 38-52, DOI 10.1112/plms/s3-46.1.38. MR684821

[4] M. Aschbacher, Finite group theory, 2nd ed., Cambridge Studies in Advanced Mathematics, vol. 10, Cambridge University Press, Cambridge, 2000. MR 1777008

[5] Olaf Düvel, On Donovan's conjecture, J. Algebra 272 (2004), no. 1, 1-26, DOI 10.1016/S00218693(03)00525-8. MR2029024

[6] Charles W. Eaton, Radha Kessar, Burkhard Külshammer, and Benjamin Sambale, 2-blocks with abelian defect groups, Adv. Math. 254 (2014), 706-735, DOI 10.1016/j.aim.2013.12.024. MR3161112

[7] Karin Erdmann, Principal blocks of groups with dihedral Sylow 2-subgroups, Comm. Algebra 5 (1977), no. 7, 665-694, DOI 10.1080/00927877708822186. MR0447391

[8] P. Fong, Simple groups with Abelian Sylow r-subgroups, unpublished manuscript (1996).

[9] Daniel Gorenstein, Finite groups, 2nd ed., Chelsea Publishing Co., New York, 1980. MR.569209

[10] Shigeo Koshitani, On the Jacobson radical of a block ideal in a finite p-solvable group for $p \geq 5$, J. Algebra 80 (1983), no. 1, 134-144, DOI 10.1016/0021-8693(83)90022-4. MR690708

[11] Shigeo Koshitani, On lower bounds for the radical of a block ideal in a finite p-solvable group, Proc. Edinburgh Math. Soc. (2) 27 (1984), no. 1, 65-71, DOI 10.1017/S0013091500022148. MR738598

[12] Shigeo Koshitani, Corrigendum: "Conjectures of Donovan and Puig for principal 3-blocks with abelian defect groups" [Comm. Algebra 31 (2003), no. 5, 2229-2243; MR1976275], Comm. Algebra 32 (2004), no. 1, 391-393, DOI 10.1081/AGB-120027931. MR2036244 
[13] Shigeo Koshitani and Burkhard Külshammer, A splitting theorem for blocks, Osaka J. Math. 33 (1996), no. 2, 343-346. MR1416052

[14] Shigeo Koshitani, Burkhard Külshammer, and Benjamin Sambale, On Loewy lengths of blocks, Math. Proc. Cambridge Philos. Soc. 156 (2014), no. 3, 555-570, DOI 10.1017/S0305004114000103. MR3181640

[15] Shigeo Koshitani and Hyohe Miyachi, Donovan conjecture and Loewy length for principal 3blocks of finite groups with elementary abelian Sylow 3-subgroup of order 9, Comm. Algebra 29 (2001), no. 10, 4509-4522, DOI 10.1081/AGB-100106771. MR.1854148

[16] Shigeo Koshitani, Jürgen Müller, and Felix Noeske, Broué's abelian defect group conjecture holds for the sporadic simple Conway group $\mathrm{Co}_{3}$, J. Algebra 348 (2011), 354-380, DOI 10.1016/j.jalgebra.2011.10.001. MR 2852247

[17] Burkhard Külshammer and Lluís Puig, Extensions of nilpotent blocks, Invent. Math. 102 (1990), no. 1, 17-71, DOI 10.1007/BF01233419. MR1069239

[18] Peter Landrock and Gerhard O. Michler, Block structure of the smallest Janko group, Math. Ann. 232 (1978), no. 3, 205-238, DOI 10.1007/BF01351427. MR0491929

[19] Peter Landrock and Gerhard O. Michler, Principal 2-blocks of the simple groups of Ree type, Trans. Amer. Math. Soc. 260 (1980), no. 1, 83-111, DOI 10.2307/1999877. MR.570780

[20] J. B. Olsson and K. Uno, Dade's conjecture for symmetric groups, J. Algebra 176 (1995), no. 2, 534-560, DOI 10.1006/jabr.1995.1258. MR.1351623

[21] Lluís Puig, Algèbres de source de certains blocs des groupes de Chevalley (French, with English summary), Astérisque 181-182 (1990), 9, 221-236. MR.1051252

[22] Atumi Watanabe, Note on a p-block of a finite group with abelian defect group, Osaka J. Math. 26 (1989), no. 4, 829-836. MR.1040427

School of Mathematics, University of Manchester, Manchester, M13 9PL, United KINGDOM

E-mail address: charles.eaton@manchester.ac.uk

School of Mathematics, University of Manchester, Manchester, M13 9PL, United KINGDOM

E-mail address: michael.livesey@manchester.ac.uk 